\title{
ULTRASONOGRAPHIC AND COMPUTED TOMOGRAPHIC FINDINGS IS DEFINITIVE IMAGING IN BILATERAL SYMMETRICAL PERIRENAL PERIPELVIC LYMPHANGIOMATOSIS: A CASE REPORT
}

Amit Kumar'1, Sanjay Suman², Sanjay Gupta ${ }^{3}$, Ranapratap Singh ${ }^{4}$

\section{HOW TO CITE THIS ARTICLE:}

Amit Kumar, Sanjay Suman, Sanjay Gupta, Ranapratap Singh. "Ultrasonographic and Computed Tomographic Findings is Definitive Imaging in Bilateral Symmetrical Perirenal Peripelvic Lymphangiomatosis: A Case Report". Journal of Evolution of Medical and Dental Sciences 2014; Vol. 3, Issue 23, June 09; Page: 6544-6549, DOI: $10.14260 /$ jemds/2014/2779

ABSTRACT: Perinephric peripelvic lymphangiomatosis is a rare benign malformation of lymphatic system; that may be confused with various forms of renal cystic diseases and urinomas. In this disorder a developmental malformation results in failure of developing lymphatic channels to establish normal communication with the rest of lymphatic systems and restrict/ failure drainage of lymphatic fluid, dilate the channels to form cystic masses that may be unilocular or multilocular and may be seen unilaterally or bilaterally. This condition presents with various signs and symptoms or can be just incidental findings which in presence of misleading clinical history may be confused with other diseases. Definitive diagnosis is possible with USG and CT scan. Here we report a case of bilateral perirenal and peripelvic involvement with a normal excretory function.

KEYWORDS: Perirenal Peripelvic Lymphangiomatosis, kidney, USG and delayed CT scan.

INTRODUCTION: Renal and perirenal lymphangiomatosis is a rare benign developmental disorder of renal lymphatic system, forming unilocular or multilocular cystic changes intrarenally or perirenally or both ${ }^{1}$.This condition may be unilateral or bilateral and it is often confused with other cystic diseases of kidney. It may be symptomatic or may be seen incidentally. CT scan with delayed images and ultrasound guided aspiration with biochemical analysis of fluid helps in diagnosis.

CASE REPORT: A 37years old woman presented with complaints of bilateral flank fullness, occasional nonspecific abdominal pain and recent onset of diarrhea for last three months. There was no significant past history of chronic illness. Physically she was of average built and on examination; she was mildly hypertensive otherwise systemic examinations were unremarkable.

Laboratory analysis revealed normal hemogram, renal functions and urine analysis. She was referred for ultrasound examination. The ultrasonography was performed with convex $3.5 \mathrm{MHz}$ probe of ultrasound unit (Nemio, Toshiba, Japan) and it revealed multi loculated, nearly symmetrical, bilateral perirenal and[Fig 1A; 1B] peripelvic cystic collections with thin septa and internal echoes.

Both kidneys were enlarged in size (right measures $13.2 \mathrm{cms}$ and left measures $14.0 \mathrm{cms}$ in length) and diffusely hyper echoic in texture (due to perinephric collection). 


\section{CASE REPORT}

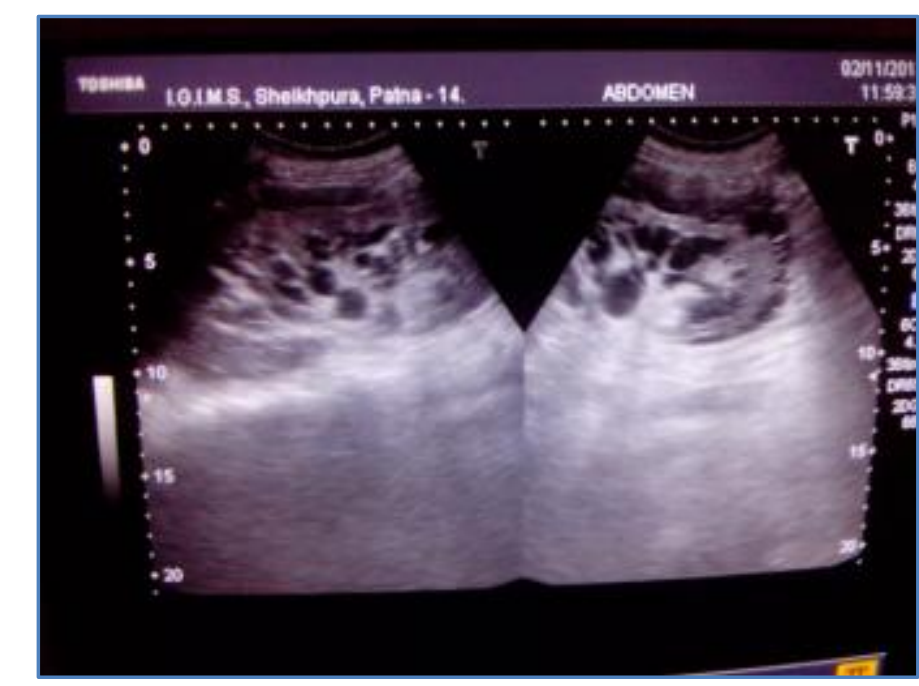

Fig 1. (A, B) :Longitudinal (A) and Transverse (B)

Ultrasound images show perirenal and peripelvic collection with septa.

MDCT of abdomen was performed on 16 detector scanner (Bright Speed GE, Milwaukee, USA). Non contrast scans showed low attenuating collection in bilateral peripelvic and perirenal regions [Fig. 2], with HU varying between 5-10. After intravenous contrast, normal cortical enhancement is seen [Fig. 3A]. The perinephric septated collections were indenting peripheral capsule-cortical surface of both kidneys. IVC was displaced antero-medially. No other abnormality was detected.

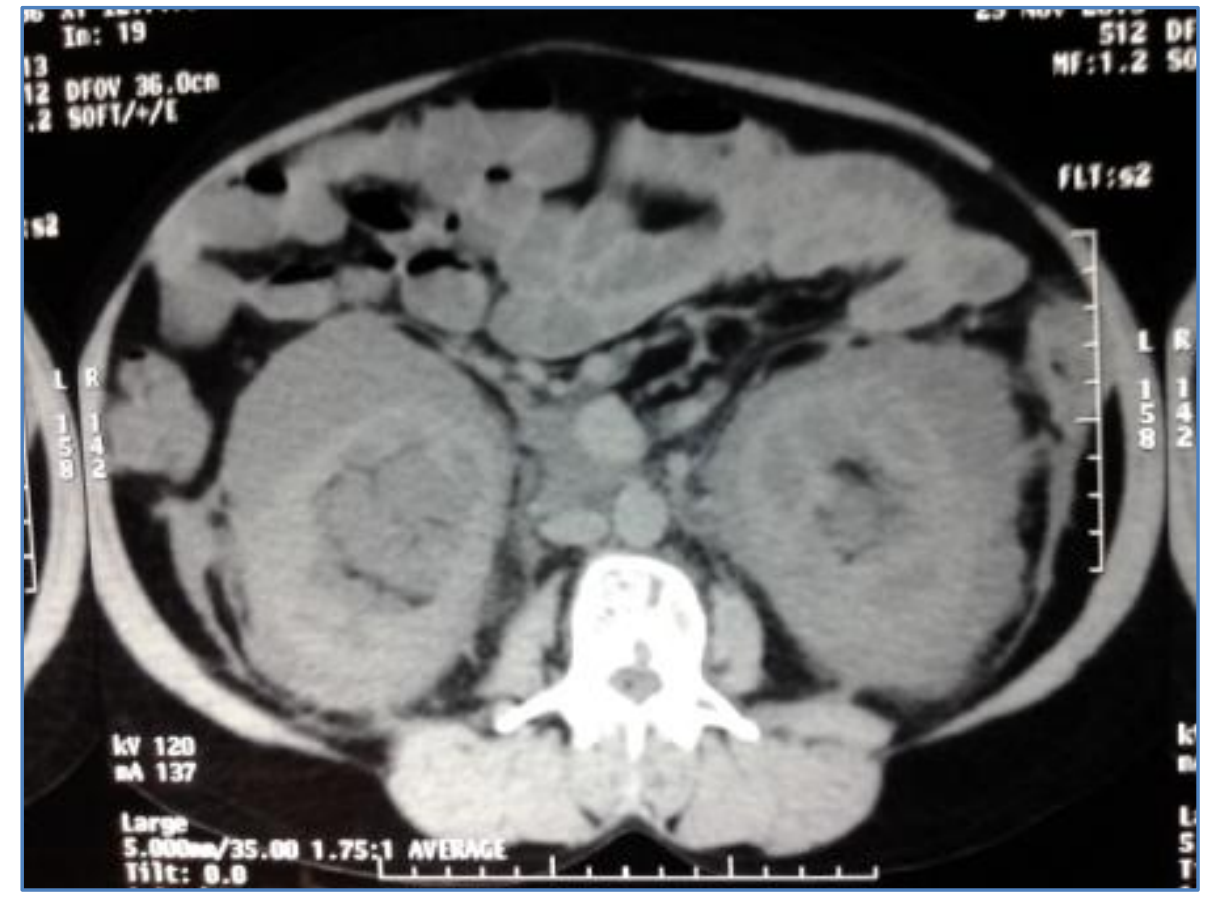

Fig. 2: NCCT axial scan shows symmetric low attenuating perirenal \& peripelvic collection 


\section{CASE REPORT}

Figure 3: CECT. Nephrogram and excretory phage shows normal cortical enhancement with non- enhancement of perinephric collections. The excretory images show splaying of the non-dilated pelvicalyceal systems.
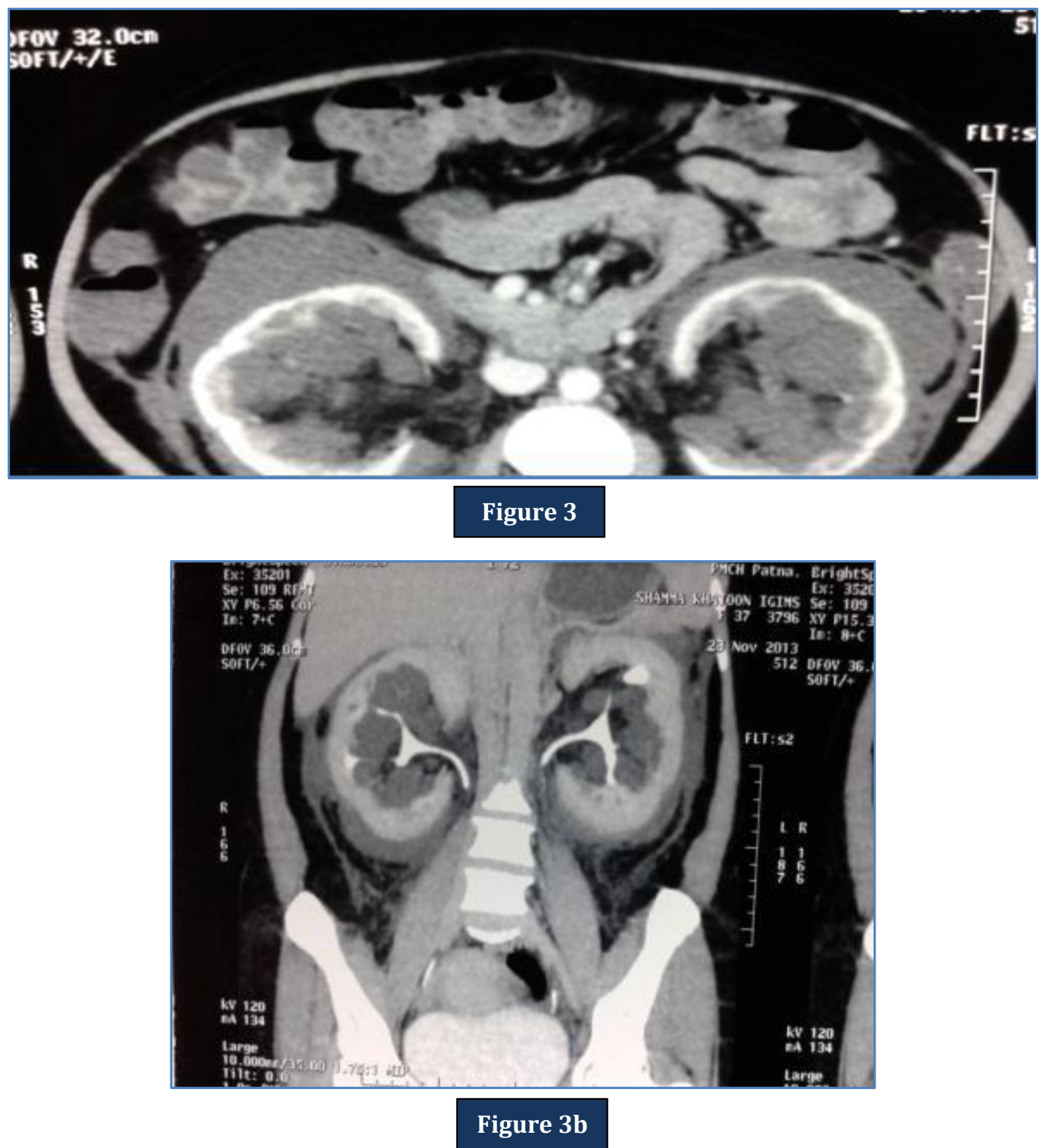

Delayed scan obtained after 15 min [Fig. 3B] shows normal excretion of contrast, but splayed pelvi-calyceal systems [Fig. 4] due to intervening fluid within sinuses. There were no extravasations of contrast in perinephric or peripelvic sinus space or no any increase in the attenuation of fluid in this space, thus ruled out communication with pelvi-calyceal systems. 


\section{CASE REPORT}

Fig. 4: CT urogram - A maximum intensity projection; coronal CT images shows splayed and non- dilated collecting systems.

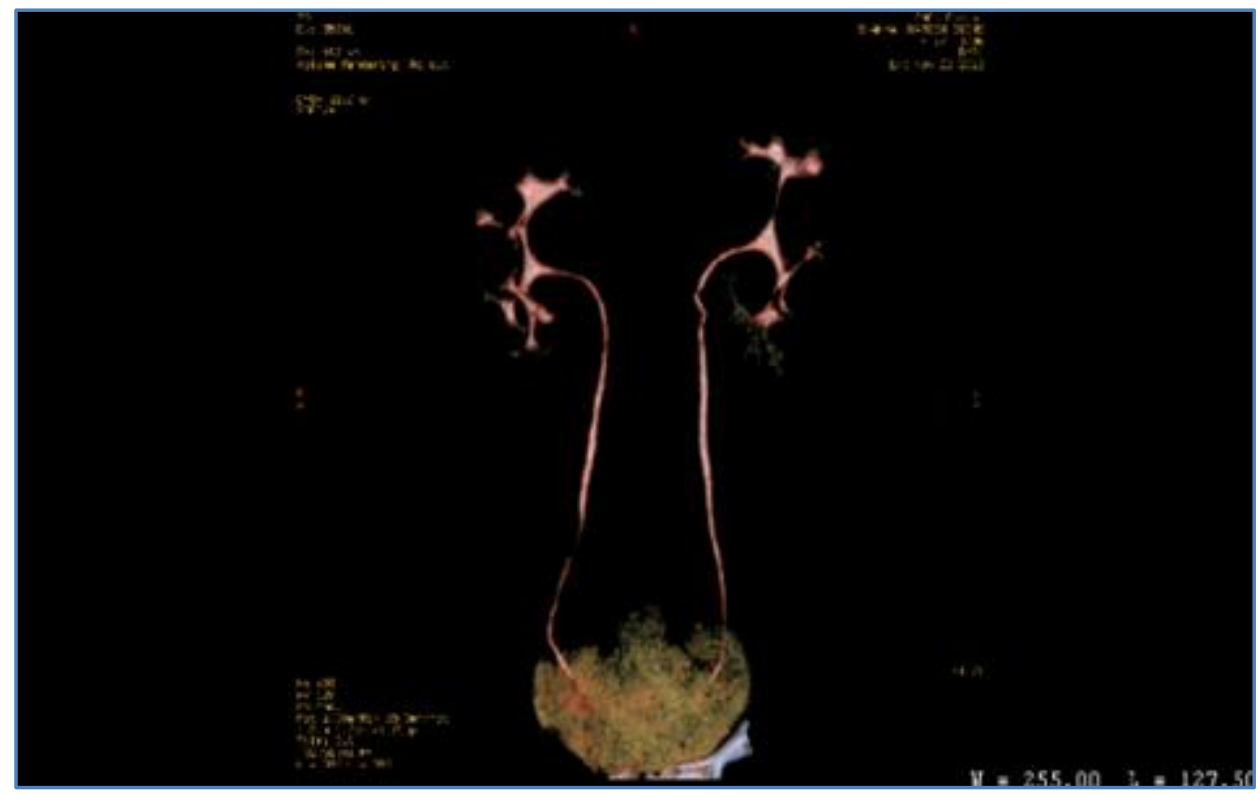

\section{Figure 4}

Ultrasound and CT findings were considered to represent perirenal and peripelvic lymphangiomatosis.

Ultrasound guided fluid aspiration was performed for the confirmation. Biochemical analysis ruled out any urinary nature of fluid. The fluid was aseptic and chylous with lymphocytes and protein and rennin specific to kidneys. It confirmed the perinephric collection as lymphatic fluid and disorder as perinephric peripelvic lymphangiomatosis. Since patient didn't have any significant sign \& symptoms related to this disorder, she was advised to come for follow up if there is any increase in blood pressure.

DISCUSSION: Lymphamgiomas are developmental in origin and common site include head and neck $(75 \%)$ and axillary region (20\%). Less than $5 \%$ occur at other sites. ${ }^{1}$

Retroperitoneal lymphangiomas accounts for $1 \%$ of all lymphangiomas with 185 cases identified in a review of international literatures. ${ }^{2}$ The reason for the development of this abnormality is failure of drainage of lymph into retroperitoneal lymphatic system. ${ }^{3}$ It may be asymptomatic or may present with nonspecific symptoms. The common complaints include flank pain, abdominal distention, hematuria, hypertension, fever and rarely impaired renal function. ${ }^{3,4}$ It is found in children as well as in adult.5,6 Familial association has also been described.7

Renal lymphangiomatosis is an extremely rare developmental disorder of lymphatic system surrounding the kidney, characterized by dilated perirenal, intrarenal, and peripelvic lymphatic systems.

On CT scan, renal lymphangioma appears as well defined fluid attenuating collection in the peri nephric/ peri pelvic space with or without septations with normal renal parenchyma.1,8,9

When intra-renal lymphatics are solely involved, renal lymphangiectasia may present as enlargement of kidney or even as a solid mass with or without cystic components. ${ }^{10}$ On MRI, they are 
hypointense on T1W images and hyperintense on T2W images. ${ }^{11}$ The diagnosis of lymphangiomatosis can be confirmed by aspiration of chylous fluid from perinephric fluid collection. ${ }^{12}$ However ultrasound and CT scan findings are generally characteristics of this entity and allow the definitive diagnosis to be made. ${ }^{13,14}$ No active treatment is required unless symptomatic or complicated.

Surgery is often required for symptom control or diagnosis. ${ }^{15}$ Complicated cases may be treated with nepherectomy, percutaneous drainage or marsupialization. ${ }^{12}$ Recurrence of symptoms with incomplete excision is possible. ${ }^{16}$ Injection of sclerotic agents such as alcohol and bleomycin into lymphangiomas has also been described as the non-surgical treatment. However, induration of the cyst and infection often complicate this procedures. ${ }^{17}$ Described complications of untreated cases include hematuria, ascites, hypertension, renal vein thrombosis and impairment of renal function. ${ }^{18}$

CONCLUSION: Perinephric lymphangioma is a rare developmental condition of kidneys however, imaging findings of this entity are generally characteristic to allow specific diagnosis. The differential diagnosis is urinoma which is differentiated by biochemical analysis of aspirated fluid. The hypertensive state of patient was supposed secondary to peripheral capsulo-cortical ischemia due to external compressive effect by chronic lymphatic collection (Page Kidney).

\section{REFERENCES:}

1. Sarno RC, Carter BL, Bank of MS. Cystic lymphangioma: CT diagnosis and thin needle aspiration. Br J Rediol. 1984; 57: 424-6.

2. Hauser H, Mischinger HJ, Beham A, Berger A, Cxrwenka H, Razmava J et al. cystic retroperitoneal lymphangiomas in adults. Eur J Surg Oncol. 1997; 23: 322-6.

3. Davidson AJ, Hartmans DS, Lymphangioma of retroperitoneum: CT and sonographic characteristics. Radiology1990; 175: 507-10.

4. Schwarz A, Lenz T, Klaen R, Offermann G, Fieldler U, Nussberger J. Hygroma renale: Pararenal lymphatic cyst associated with rennin dependent hypertension (page kidney): case report on bilateral cysts and successful therapy by marsupialization. J Urol 1993; 150: 953-7.

5. Murray K K, Mclellan GL, Renal pelvic lymphangiectasia: appearance at CT. Radiology 1991; 180: 455-6.

6. Blumhagen ID, Wood BJ, Rosenbavm DM, Sonographic evaluation of abdominal lymphangioma in children J Ultrasound Med 1987; 6: 487-95.

7. Meredit WT. Levine EAhlstom NG, Grantham II. Exacerbation of familial renal lymphangiomatosis during pregnancy, AJR Am J Roentgenol 1988; 151: 965-6.

8. Rreidahl WH, Mendelson RM, Retroperitoneal lymphangioma, Australas Radiol 1995; 39: 187-9

9. Ramseyer LT, case 34: renal lymphangiectasia. Radiolosy 2001; 219: 442-4.

10. Lim JK, Ahn JH, Kim KR, Cho KS, Renal lymphangioma manifested as a solid mass on ultrasonography and computed tomography. J ultrasound med 2002; 21: 203-6.

11. Riehl J, Schmitt H, Schofer L, Schneider B, Sieberth HG, retroperitoneal lymphangiectasia associated with B/L renal vein thrombosis. Nephrol dial Transplant 1997; 12: 1701-3.

12. Murray KK, McLellan GL. Renal peripelvic lymphangiectasia: Appearance at CT. Radiology. 1991; 180: 455-6.

13. Meredith WT, Levine E, Ahlstom NG, Grantham JJ. Exacerbation of familial renal lymphangiomatosis during pregnancy. AJR Am J Roentgenol. 1988; 151: 965-6. 


\section{CASE REPORT}

14. Chait D, Yonkers AJ, Beddoe GM, Yarington CT Jr, Management of cystic hygroma. Surge Gyne co/ obstet 1974; 139: 55-8.

15. Burkett JS, Pickleman J, the rationale for surgical treatment of mesenteric \& retroperitoneal cyst. Am Surg 1994; 60: 432-5.

16. Tanigowa N, Shimomatsuya $\mathrm{T}$, Takahashi $\mathrm{K}$, Inomata $\mathrm{Y}$, Tanaka K, Satomuva K, eral. Treatment of cystic lymphangioma and hygroma with the use of Bleomycin fat emulsion. Cancer 1987; 60: 741-9.

17. Riehl J, Schmitt H, Schafer L, Schneider B, Sieberth G. Retroperitoneal lymphangiectasia associated with bilateral renal vein thrombosis. Nephrol Dial Transplant. 1997; 12: 1701-3.

18. Chang MS, Yang DM, Yoon MH, Kim HS, Jin W, Lee JB. Peri pelvic \& Peri renal lymphangiectasia and bilateral renal vein thrombosis: A case report. J Korean Radiol SOC 2000; 43: 91-4.

\section{AUTHORS:}

1. Amit Kumar

2. Sanjay Suman

3. Sanjay Gupta

4. Ranapratap Singh

\section{PARTICULARS OF CONTRIBUTORS:}

1. Senior Resident, Department of Radiology, IGIMS, Patna.

2. Associate professor, Department of Radiology, IGIMS, Patna.

3. M.ch. Trainee, Department of Urology, IGIMS, Patna.

4. M.ch. Trainee, Department of Urology, IGIMS, Patna.

\section{NAME ADDRESS EMAIL ID OF THE CORRESPONDING AUTHOR: \\ Dr. Amit Kumar, \\ \#C-28, Anand Vihar Colony, \\ Road No. 2, Ambedkar Path, \\ Jagdeo Path, Patna-14. \\ E-mail: amitmd2008@gmail.com}

Date of Submission: 08/05/2014. Date of Peer Review: 09/05/2014. Date of Acceptance: 30/05/2014. Date of Publishing: 09/06/2014. 